\title{
Spermatogonial stem cell biology in the bull: development of isolation, culture, and transplantation methodologies and their potential impacts on cattle production
}

\author{
JM Oatley \\ Center for Reproductive Biology and Health, Department of Dairy and Animal Science, The \\ Pennsylvania State University, University Park, PA 16802
}

Widespread adoption of artificial insemination as a breeding practice has allowed for expanded use of desirable genetics from specific sires and greatly influenced production traits in dairy cattle populations worldwide. In fact, the average dairy cow in the US in 2009 produced 4.5 times more milk than in 1940 when commercialization of artificial insemination began. While many factors have contributed to this rapid increase in levels of milk production, genetic gain through expanded utilization of germlines from specific sires has been a major contribution. In comparison, use of artificial insemination in beef cattle populations has been limited due to challenges with implementing intensive management strategies required for success. Thus, there is need for alternative reproductive tools to expand use of desirable male genetics in the beef cattle industry. The process of sperm production, termed spermatogenesis, is supported by a tissuespecific stem cell population referred to as spermatogonial stem cells (SSCs). These unique cells have the capacity for infinite self-renewal and long-term regeneration of spermatogenesis following transplantation. In rodents, methods for isolating, culturing, and transplanting SSCs have been devised. For beef cattle, transplanting SSCs isolated from a donor male into the testes of recipient males in which donor-derived spermatogenesis occurs and offspring with donor genetics are produced from natural breeding has great potential as an alternative to artificial insemination. This potential reproductive strategy would allow for expansive use of genetics from desirable sires that overcomes the logistical challenges of artificial insemination. Translation of the methods devised for rodents to cattle is at the forefront of development. Devising means for isolating an SSC-enriched cell fraction from donor testes and identifying conditions that support long-term maintenance and proliferation of bovine SSCs in vitro are two tools that would greatly accelerate the pace at which transplantation will become a commercially viable option for cattle industries. Recent studies showed that expression of THY1 by SSCs is a conserved phenotype between rodents and cattle, and selection of the THY1 + fraction from 
donor testes can be used for isolating an SSC-enriched germ cell population. In addition, the conditions devised for expanding the number of rodent SSCS in vitro continues to serve as the basis for developing conditions that support bovine SSCS. With these tools in hand major advances in developing implementable reproductive tools with SSCs for commercial cattle production will be made in the coming decade.

\section{Introduction}

Utilization of desirable genetics is a key aspect of animal agriculture, allowing for rapid expansion of phenotypic improvement in livestock populations. Currently, the most effective means to enhance efficiency of food animal production and improve product quality is through introduction and expansion of specific genetics. In most agriculturally important animal populations the majority of genetic gain is made through the male germline. Thus, male reproductive efficiency is an essential component of livestock production and devising means to expand availability of male genetics can have major economic impacts to the producer and consumer. Spermatozoa are the cell by which genetics of males are passed to the next generation and are a major target for preserving the germline after death and expanding the utilization of superior genetics in cattle populations. One major avenue that sperm have been utilized in cattle production is with artificial insemination (Al). Since its commercialization in the 1940's, Al has had major impacts on cattle production over the last 70 years, especially with genetic improvement of dairy cattle herds (Foote 2002). Widespread adoption of Al in the dairy industry to capitalize on specific genetics has been a main factor for generation of a cattle population in 2009 that produces on average 4.5 times more milk per cow than in 1940 (National Agricultural Statistics Service). It has been estimated thatapproximately $80 \%$ of dairy cattle in the US are bred by $\mathrm{Al}$ compared to only approximately $5 \%$ of beef cattle, resulting in lost opportunities for genetic gains in this industry (Geary et al., 1998; Perry et al. 2002). Effective implementation of an $\mathrm{Al}$ program requires intensive management, posing a practical limitation for beef cattle enterprises in which the majority of animals are typically managed in range conditions where intensive management is challenging. Thus, there is need for devising alternative reproductive strategies that allow for expansion and utilization of the germline from genetically desirable sires in beef cattle populations.

\section{Spermatogenesis and Spermatogonial Stem Cells (SSCs)}

Spermatogenesis is the process by which millions of sperm are generated per day in the testis, commencing at puberty and persisting throughout old age in males (Sharpe, 1994). At the foundation of this process are the spermatogonial stem cells (SSCS) which undergo both selfrenewal and differentiation. The process of self-renewal maintains a pool of SSCs throughout the life of a male and provides the basis for continual male fertility. Initiation of sperm production occurs when SSC differentiation results in production of daughter progeny, termed $A_{\text {paried }}\left(A_{p r}\right)$ spermatogonia, which are committed to differentiation rather than self-renewal (de Rooij and Russell, 2000). The $A_{p r}$ spermatogonia then undergo a series of mitotic cell divisions becoming $A_{\text {aligned }}\left(A_{\text {all }}\right)$ spermatogonia and give rise to differentiating type $A$ spermatogonia which undergo another series of amplifying mitotic divisions. These differentiating A spermatogonia mature into intermediate and type B spermatogonia which enter meiosis becoming primary and secondary spermatocytes, and eventually haploid spermatids are produced which undergo a transforma- 
tion into spermatozoa (Russell et al., 1990). Collectively, the SSCs (also termed $A_{\text {single }}$ or $A_{s}$ ), $A_{p r}$ and $A_{d \mid}$ germ cells are referred to as the undifferentiated spermatogonial population and share many phenotypic and molecular characteristics (Oatley and Brinster 2008). Similar to other tissue-specific stem cell populations SSCs are rare within their residing tissue, constituting an estimated $0.03 \%$ of the total testicular cell population in adult mice (Tegelenbosch and de Rooij, 1993). While the SSC population has not been studied in male livestock to any great detail, spermatogenesis is conserved among mammalian species and characteristics defined for SSCs in rodents are likely conserved in higher order mammals such as cattle (Sharpe, 1994). Due to the extreme rarity of SSCs studying and isolating these cells is a major challenge which has hampered the pace at which the biology of these cells has been defined.

\section{Conserved molecular characteristics of SSCS}

Currently, molecular markers specific for SSCs that distinguish them from the other undifferentiated spermatogonia (i.e. $A_{p r}$ and $A_{a l}$ ) have not been described. The only unequivocal measure of SSCs is a functional capacity to regenerate and maintain spermatogenesis. Nonetheless, expression of several molecular markers that distinguish undifferentiated spermatogonia, including SSCs, from differentiating spermatogonia have been identified. Studies in the mouse showed that expression of the transcriptional repressor promyelocytic leukaemia zinc finger (PLZF) is restricted to the undifferentiated spermatogonial population (Buaas et al., 2004; Costoya et al., 2004); whereas, expression of C-KIT is restricted to differentiating spermatogonia (Yoshinaga et al., 1991). Recent studies by Reding et al. (2010) with cattle and Lou et al. (2009) with pigs showed that expression of PLZF is localized to undifferentiated spermatogonia, suggesting at least partial conservation of phenotypic and molecular characteristics of spermatogonia in rodents and livestock. Those same studies showed that expression of ubiquitin carboxyl-terminal esterase L1 (UCHL1) is a general marker of type A spermatogonia in cattle and pigs (Lou et al,, 2009; Reding et al., 2010). Currently, localization of c-KIT expression by spermatogonia in livestock has not been reported in detail but will be important to characterize for understanding how the SSC population is defined in agriculturally important animals such as bulls. Overall, use of PLZF, UCHL1, and C-KIT as markers provides a tool to better define different spermatogonial populations including SSCs.

\section{Niche factors regulating SSC fate decisions}

Similar to other tissue-specific stem cell populations found in mammals maintenance of a pool of SSCS and regulation of their biological activities is supported by a niche microenvironment (Oatley and Brinster, 2008). This specialized environment is made up of both growth factors and microarchitecture provided by niche support cells. Coordination between the specific growth factors secreted by support cells and expression of receptors by SSCs is essential for maintenance and function of the SSC pool. Thus, defining the support cell populations and their specific contributions to the niche is important given that disruption of these contributions will impair male fertility. The majority of understanding about growth factor components of the SSC niche has been defined using the mouse as a model and conservation of those mechanisms in livestock are only just beginning to be explored (Reding et al., 2010). For mouse SSCs, specific cytokines identified as components of the niche include glilal cell line-derived neurotrophic factor (GDNF), fibroblast growth factor 2 (FGF2), and colony stimulating factor 1 (CSF-1). Reduced expression of GDNF in mice with one null allele results in rapid decline of male fertility 
following puberty that is attributed to formation of seminiferous tubules devoid of germ cells, a condition referred to as Sertoli-cell-only (Meng et al., 2000). Also, overexpression of GDNF in mice causes accumulation of the undifferentiated spermatogonial population culminating in formation of germ cell tumors (Meng et al., 2000). These in vivo studies suggested that GDNF plays a key role in regulating the fate decisions of SSCs in mammalian testes. Subsequent studies by Kubota et al. (2004a) also utilizing the mouse showed that GDNF could promote long-term survival and expansion of SSC numbers in a serum-free chemically defined environment in vitro, solidifying its role as a key regulator of SSC self-renewal. Because of their intimate association with germ cells and known role as "nurse" cells for spermatogenesis, Sertoli cells have been regarded as the main support cell population for SSC niches. Indeed, Sertoli cells were shown to express GDNF in response to stimulation from follicle stimulating hormone (FSH) which is a key regulator of their functional capacity to support spermatogenesis (Tadokoro et al., 2002). Additional studies by Kubota et al. (2004a) showed that FGF2 supplementation enhances GDNF-induced proliferation of mouse SSCs in vitro but is unable to sustain SSCs as the sole growth factor supplement. Similar to GDNF, production of FGF2 has also been tied to Sertoli cells in the mouse testis (Mullaney and Skinner, 1992). A third factor that contributes to the milieu of extrinsic stimuli that influence SSC self-renewal is CSF-1. In vitro, exposure to soluble CSF-1 enhances GDNF influence on SSC self-renewal specifically and expression of this molecule in vivo is localized to Leydig cells (Oatley et al., 2009). Collectively, these findings implicate both Sertoli and Leydig cells as contributors to the SSC niche in mouse testes. While several key growth factors that regulate SSC self-renewal and survival in vitro have been identified, their rate of proliferation is slow (estimated to be approximately 6 days) and a pure population cannot be maintained suggesting that additional factors affecting their fate decisions have yet to be identified. Unfortunately, expression of GDNF, FGF2, or CSF-1 in the testes of livestock animals has not been reported but will be an area of investigation in the coming decade as more emphasis is placed on translating findings from rodents studies on SSC biology to higher order mammals.

\section{SSC transplantation}

A key aspect of SSC biology is their ability for efficient transplantation and regeneration of spermatogenesis. This capability is especially important for altering the genetic makeup of animal populations given that SSCS are the only permanent self-renewing cell type in mammals that contribute genetic information to the next generation. In 1994, Brinster and colleagues reported the regeneration of donor-derived spermatogenesis following microinjection of a single cell suspension of testicular cells isolated from a donor mouse into the testes of recipient mice and offspring containing donor genetics were generated after mating (Brinster and Zimmerman, 1994; Brinster and Avarbock, 1994). These pioneering studies were the first to prove existence of a stem cell population within mammalian testes and opened a new field of study in male reproductive biology. Stem cells are defined by an ability to maintain tissue homeostasis and regenerate long-term tissue function following toxic damage that depletes the tissue of differentiated cells or following transplantation. For the testis, long-term regeneration of spermatogenesis is the defining feature of testicular stem cells. Because the undifferentiated spermatogonial population represents the foundation of spermatogenesis it is believed to contain the testicular stem cells and this regenerating cell population was labeled as spermatogonial stem cells or SSCs. Ever since the first reports of transplantation success, several groups have aimed to extend this methodology to other mammals including humans and livestock. 
For cattle, the SSC transplantation method has the potential to provide a novel avenue to (1) immortalize the germline of genetically desirable sires, (2) efficiently expand utilization of desirable male genetics, and (3) provide an efficient means for generation of transgenic cattle. These possibilities are especially applicable to the beef cattle industry where use of Al has historically been limited due to impracticality. One potentially valuable application would be transplantation of SSCs from a desirable donor bull into the testes of a group of recipient bulls in which donor-derived spermatogenesis would occur and the recipients breed by natural service to generate offspring with the donor genetics (Figure 1). This potential reproductive tool would overcome the logistical difficulties associated with implementation of $\mathrm{Al}$, allowing for efficient utilization of desirable male genetics. As a result, genetic gain in beef cattle populations could be enhanced leading to more efficient production of meat and fiber for human consumption. While the potentials are great, translation of SSC transplantation methods devised for mice to cattle has been limited. In mice, isolation of an SSC-enriched fraction from the total testicular cell population for injection into recipient testes has dramatically enhanced the efficiency of SSC transplantation success, and this principal has recently begun to be developed for bulls (Reding et al., 2010).

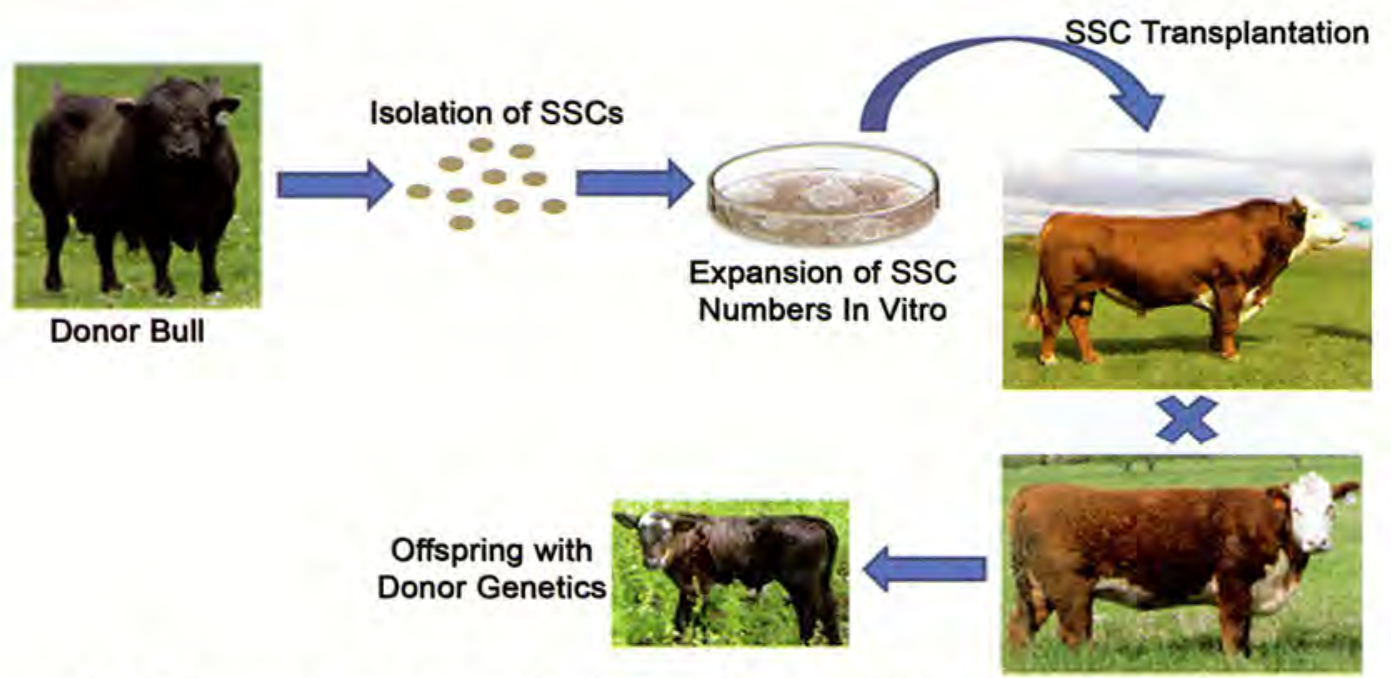

Fig. 1. Scenario for application of SSC transplantation in beef cattle. Isolation of SSCs from the testis of a genetically desirable bull followed by a period of in vitro maintenance that supports their proliferation would provide a source of donor SSCs for transplantation into recipient bulls. Donor-derived spermatogenesis would then occur within recipient testes and these bulls could breed by natural service to generate offspring containing donor genetics. This potential application would provide a novel reproductive tool for expanding the use of desirable male genetics in the beef cattle industry.

Due to rarity of SSCs in mammalian testes extensive regeneration of donor-derived spermatogenesis within recipient testes is limited without enrichment prior to transplantation. This limitation has prompted the search for cell surface markers that could be used with cell sorting technologies such as fluorescent activated cell sorting (FACS) or magnetic activated cell sorting (MACS) to isolate an SSC enriched fraction for transplantation. The mouse has been predominately used as a model to identify cell surface markers of mammalian SSCs. However, recent progress has been made in translating findings in the mouse to bulls (Reding et al., 2010). The 
first reported markers of mouse SSCs were $\alpha 6$-integrin and $\beta 1$-integrin which resulted in 8 -fold and 4-fold enrichment for SSCs following transplantation, respectively (Shinohara et al., 1999). To date, the greatest enrichment for mouse SSCs based on a single cell surface protein is by selection of the THY1 + cell fraction (Oatley and Brinster, 2008). In adult mouse testes, the THY1 + cell fraction is enriched 30 -fold compared to the unselected total testis cell population (Kubota et al., 2004b). Logically, because GDNF is an essential growth factor promoting SSC self-renewal selection of spermatogonia expressing the associated receptors, c-RET and GFR $\alpha 1$, should result in enrichment for SSCs. Unfortunately, both the c-RET + and GFR $\alpha 1+$ testis cell fractions from adult mice are depleted of SSCs indicating these are not effective markers for isolation of stem cells (Ebata et al., 2005). Other surface proteins identified as markers for rodent SSCs include CD9 (Kanatsu-Shinohara et al,, 2004), Ep-CAM (Ryu et al., 2005), and GPR125 (Seandel et al., 2007), but none have shown the level SSC enrichment found with THY1 (Oatley and Brinster 2008).

Conservation of cell surface phenotype for SSCs among mammals is just beginning to be explored. Recent studies by Reding et al. (2010) showed that THY1 is a conserved maker of SSCs in bulls and that selection of the THY1 + cell fraction by MACS results in isolation of an SSC-enriched population from testes of pre-pubertal bulls. The development of SSC transplantation methods in mice was greatly advanced by identification of SSC surface markers that allowed for their isolation (Oatley and Brinster 2006; Oatley and Brinster 2008). With the recent progress in identify markers of SSCs in bulls the pace at which development of SSC transplantation in cattle is sure to increase.

Even without selection of an SSC enriched population for injection several studies have attempted transplantation in bulls utilizing unselected testis cell populations (Izadayar et al., 2003; Herrid et al., 2006; Stockwell et al., 2009; ). While none of these studies have provided definitive results of success, important information has been gleaned about feasibility of the testis injection procedure. The greatest challenge for determining success of SSC transplantation in bulls is unequivocal identification of donor-derived spermatogenesis. In rodents, this has been achieved through the use of naturally sterile recipients in which any regeneration of spermatogenesis is donor-derived or with donors that express a marker transgene (e.g. LacZ or GFP) within the germline (Oatley and Brinster 2006). Izadayar et al. (2003) performed homologous and autologous germ cell transplantation with 5 month old Holstein bulls following localized irradiation of recipient testes to deplete endogenous spermatogenesis. At 2.5 months after injection of donor germ cells cross-sections of recipient testes were examined for spermatogenesis histologically and those receiving donor cells contained a greater proportion of seminiferous tubules with both spermatogonia and complete spermatogenesis (Izadayar et al., 2003). Unfortunately, the donor cells were not labeled for detection after transplantation and spermatogenesis within recipient seminiferous tubules could not be unequivocally proven to be donor-derived. Also, by definition transplanted germ cells must support long-term regeneration of spermatogenesis (i.e. several rounds of spermatozoa production) to be considered SSCs but the timeframe of analysis employed by Izadayar et al. (2003) only examined spermatogenesis within recipient bulls after one round of spermatogenesis. Moreover, in mice germ cell development from spermatogonia to spermatozoa occurs over a 35 day period but the first round of spermatogenesis from transplanted SSCs is delayed requiring at least 1.5 cycles or $\sim 52$ days (Nagano et al., 1999). In bulls, spermatogenesis is approximately 61 days; thus, identification of donor-derived spermatogenesis after 2.5 months ( $\sim 75$ days) reported by Izadayar et al. (2003) suggests regeneration from transplanted bovine SSC.S is accelerated compared to the mouse. Using detectable differences in microsatellite markers Stockwell et al. (2009) detected donor spermatozoa within the ejaculate of recipient bulls 52-98 weeks after injection of germ 
cells, but their appearance declined over time. These results indicated that the donor-derived spermatozoa were not a result of SSC colonization given that long-term regeneration of spermatogenesis was not achieved. Even with these areas of contention for success of bovine SSC transplantation, previous studies have provided the framework for refinement in the future to develop this technique as a reproductive tool for expanding utilization of the male germline.

\section{Long-term culture of SSCs}

In vitro maintenance of SSCs for long periods of time in conditions that support their selfrenewal is a key aspect for developing reproductive technologies that utilize these cells to immortalize and expand the use of germlines from desirable males. The rarity of SSCs even if isolated from donor testes is a limiting factor for success of transplantation into recipients and widespread utilization of this technology. The potential for infinite expansion of SSCs in vitro through support of self-renewal would provide millions of these cells for transplantation into hundreds of recipient bulls. Moreover, when combined with cryopreservation methodologies the germline of specific males could be immortalized. These possibilities have advantage over sperm cryopreservation as the standard for germline preservation after death given that sperm are a non renewable resource without the associated SSC population. To date, techniques for long-term maintenance and expansion of SSCs are only available for mice (Kubota et al., 2004a; Oatley and Brinster 2006), rats (Ryu et al., 2005), and hamsters (Kanatsu-Shinohara et al., 2007). Previous studies with cattle have resulted in short-term proliferation of SSCs, but long-term maintenance has not been achieved (Oatley et al., 2004a; Oatley et al., 2004b; Aponte et al., 2005). Additionally, culture of SSCs from other livestock species has not been reported. However, utilizing the mouse system as a basis our lab has made strides in refining culture conditions for maintenance of bovine SSCs (Figure 2).
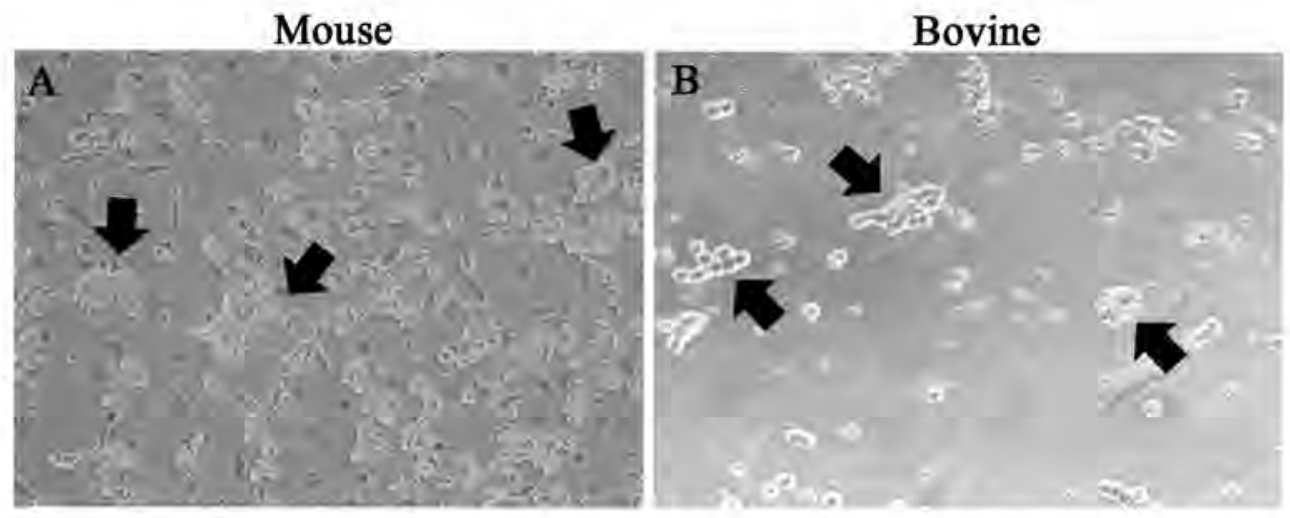

Fig. 2. Formation of germ cell clumps (arrows) in cultures of THY1 + cells isolated from the testes of mice (A) and bulls (B). Cultures were maintained in serum-free chemically defined media with either mouse serum-free nutrient supplement (mSFM, Kubota et al., 2004a) for mouse cells or StemPro ${ }^{\oplus}$ serum-replacement for bovine cells. Also, the media was supplemented with the growth factors GDNF and FGF2. The germ cells loosely attach to an underlying feeder cell monolayer composed of STO cells for mouse cultures and bovine embryonic fibroblasts (BEFs) for bovine cultures. The clumps appear morphologically similar between the two species and mouse cells are proven to contain SSCs. Thus, the formation of these germ cell clumps from bovine testes may contain bona fide SSCs. 
For rodents, maintenance and proliferation of SSCs in vitro requires mitotically inactive feeder cell monolayers and optimized serum-free medium with specific nutrient and growth factor supplementations. Feeder cells derived from mouse embryos have proven the most effective at supporting self-renewal of rodent SSCs for several months in vitro (Oatley and Brinster 2006). Immortalized STO (mouse embryonic fibroblast) feeder cell monolayers support expansion of both mouse (Kubota et al., 2004a) and rat (Ryu et al., 2005) SSC numbers for greater than 5 months in culture. In addition, primary mouse embryonic fibroblasts (MEF) support long-term self-renewal of mouse gonocytes (precursors to SSCs) in vitro (Kanatsu-Shinohara et al,, 2003). With bulls, primary bovine embryonic fibroblasts (BEF) have been utilized as feeders and support short-term expansion of bovine SSCs (Oatley et al., 2004b). When maintained in complex serum containing medium SSC numbers increased over a 7 day period with BEF feeders, but rapidly declined after 14 days suggesting that long-term self-renewal could not be supported (Oatley et al., 2004b).

In addition to feeder cell monolayers, expansion of rodent SSC numbers in vitro is effectively supported in serum-free medium conditions (Kubota et al., 2004a; Ryu et al., 2005; KanatsuShinohara et al., 2005). In contrast, several cell types, including embryonic stem cells, require the addition of fetal bovine serum (FBS) in basal media to support growth. The complexity of nutrients in FBS preferentially supports proliferation of rapidly dividing cells. Because SSCS divide relatively slowly (Kubota et al., 2004a), other rapidly dividing cell types such as testicular fibroblasts outgrow SSCs when cultured in serum-containing medium resulting in loss of SSCs over time. Also, FBS appears to have toxic effects on mouse and rat SSCs in culture (Kubota et al., 2004a; Ryu et al., 2005). Previous attempts at culturing bovine SSCs have included FBS in basal medium (Dobrinski et al., 2001; Oatley et al, 2002; Oatley et al , 2004a; Oatley et al., 2004b; Aponte et al., 2005). In those studies, short-term expansion of bovine SSCs was observed followed by rapid decline of SSC numbers at which time fibroblast take-over was observed, likely impairing SSC proliferation and survival (Dobrinski et al., 2001; Oately et al., 2002; Oatley et al., 2004b).

For rodent SSCs, a defined serum-free condition composed of Minimal Essential Media Alpha $(M E M \alpha)$ as the base medium with specific nutrient supplementation supports SSC survival and proliferation for extended periods of time (Nagano et al., 2003; Kubota et al., 2004a; Kubota et al., 2004b). Mouse SSC culture conditions developed by Kubota et al. (2004a) utilized a chemically-defined serum-free supplement (mSFM). Extension of this system to support rat SSCs required enrichment of specific components of mSFM leading to creation of a rat serum-free supplement (rSFM; Ryu et al., 2005). In both these supplements each component is added at specific concentrations, providing consistent results because media conditions can be acutely controlled between batches. In less defined conditions, supplementation with commercially produced StemPro serum-replacement (Invitrogen; Carlsbad, CA) also supports long-term self-renewing expansion of mouse SSCs (Kanatsu-Shinohara et al., 2005). In those conditions, supplement components are proprietary and batch-to-batch variation effects reproducibility of SSC proliferation in vitro. To date, the effects of any basal medium and serum-free condition with nutrient supplementation that supports rodent SSCs in vitro has not been reported with livestock SSCs.

SSC self-renewing proliferation in serum-free conditions is limited without the addition of specific growth factors. Addition of soluble GDNF is essential for expansion of mouse, rat, and hamster SSC numbers when cultured in defined media conditions (Kubota et al., 2004a; Ryu et al., 2005; Kanatsu-Shinohara et al., 2007). Importantly, preliminary studies with bovine germ cells showed that addition of GDNF to complex culture medium enhanced short-term expansion of SSCs over a 14-day period (Oatley et al., 2004b). Studies with the mouse showed that insulin-like growth factor 1 (IGF-1), epidermal growth factor (EGF), FGF2, CSF-1, and leuke- 
mia inhibitory factor (LIF) enhance GDNF influence on SSC maintenance and proliferation in serum-free conditions (Kubota et al., 2004a; Kubota et al., 2004b; Kanatsu-Shinohara et al., 2005; Kanatsu-Shinohara et al., 2007). In serum-free medium with specific nutrient (e.g. mSFM, rSFM, or StemPro ${ }^{(2)}$ and growth factor supplements SSCs grow as three dimensional clumps of germ cells loosely attached to the feeder cell monolayer (see Figure 2). Similar generation of germ cell clumps in vitro from bovine testis cells has not been reported. Instead, studies by Oatley et al. (2004b) and Aponte et al. (2005 and 2008) have shown maintenance of cells with a fibroblast-like morphology indicating either bovine SSC morphology in vitro is distinctly different from rodents or the cultures did not contain bona fide SSCs.

Translation of conditions that support proliferation of rodent SSCs in vitro to SSCs of other mammals has been challenging and not reported to date in peer reviewed literature. One aid in establishing mouse SSC cultures has been isolation of an SSC-enriched cell fraction from donor testes for initial setup of the culture. Elimination of the non SSC components of a testicular cell population especially the rapidly dividing somatic cells is advantageous for growth of SSCs in vitro. For development of mouse SSC cultures the isolated THY $1+$ cell fraction was utilized and is commonly used to effectively maintain and expand the number of SSCs in serum-free chemically defined conditions. Recent studies by Reding et al. (2010) showed that the THY1+ cell fraction from bull testes is enriched for SSCs indicating conserved characteristics for these cells among mammalian species. Thus, it is likely that conditions supporting proliferation of mouse SSCs in vitro can serve as a basis for tailoring conditions that support SSCs of other mammals including cattle. In fact, our recent studies have tested the mouse condition of MEM $\alpha$ base medium with StemPro ${ }^{\circledR}$, GDNF, and FGF2 supplementation with BEF feeders for support of bovine THY1 + SSCS and observed the formation of germ cell clumps resembling those that develop in mouse cultures (Figure 2). For the first time, these cultures may represent maintenance of bovine SSCs in vitro. Future work will involve refining these conditions to support long-term proliferation that can serve as a source of donor SSCs for further development of transplantation methods in bulls.

\section{Conclusions}

Major advances in genetic gain of cattle populations can be made with reproductive tools that expand the availability of germlines from desirable males. While Al has been widely used in dairy cattle to achieve this and resulted in major improvements of milk production over the last 70 years, beef cattle populations have lost opportunities for genetic improvement because of the impracticality needed for implementation of Al programs. The unique functions of the SSC population for self-renewal and regeneration of continual spermatogenesis provides a novel alternative to $\mathrm{Al}$ for expanding the utilization of desirable male genetics in the beef cattle industry. Reproductive tools based on SSCs such as transplantation are at the forefront of being developed in cattle. In rodents, progression of techniques for isolation, culture, and transplantation of SSCs spanned greater than a decade of research. While several studies have reported limited development of culture conditions for bovine SSC S and transplantation between donor and recipient bulls, none have provided definitive evidence of success that is close to commercialization or useful as a tool in livestock production. One of the major limitations has been lack of methods to isolate an SSC-enriched cell fraction from bull testes. Recently, Reding et al. (2010) reported this capability based on selection of THY1 + cells, adding a key tool for further developing culture and transplantation methods for bulls. Major advances in developing these techniques as usable tools in the cattle industry will be made in the coming decade. 


\section{References}

Aponte PM, Soda T, Teerds KJ, Mizrak SC, van de Kant HJ \& de Rooij DG 2008 Propagation of bovine spermatogonial stem cells in vitro. Reproduction 136 543-557.

Aponte PM, van Bragt MP, de Rooij DG \& van Pelt AM 2005 Spermatogonial stem cells: characteristics and experimental possibilities. Acta Pathologica, Microbiologica et Immunologica Scandinavica 113(11-12) 727-742.

Brinster RL \& Avarbock MR 1994 Germline transmission of donor haplotype following spermatogonial transplantation. Proceedings of the National Academy of Sciences 91 11303-11307.

Brinster RL \& Zimmermann JW 1994 Spermatogenesis following male germ-cell transplantation. Proceedings of the National Academy of Sciences $\mathbf{9 1}$ 11298-11302.

Buaas FW, Kirsh AL, Sharma M, McLean DJ, Morris JL, Griswold MD, de Rooij DG \& Braun RE 2004 Plzf is required in adult male germ cells for stem cell selfrenewal. Nature Genetics 36(6) 647-652.

Costoya JA, Hobbs RM, Barna M, Cattoretti G, Manova K, Sukhwani M, Orwig KE, Wolgemuth DJ \& Pandolfi PP 2004 Essential role of Plzf in maintenance of spermatogonial stem cells. Nature Genetics 36(6) $653-652$.

de Rooij DG \& Russell L.D 2000 All you wanted to know about spermatogonia but were afraid to ask. Journal of Andrology $21776-798$.

Dobrinski I, Ogawa T, Avarbock MR \& Brinster RL 2001 Effect of the $\mathrm{GnRH}$-agonist leuprolide on colonization of recipient testes by donor spermatogonial stem cells after transplantation in mice. Tissue and Cell 33(2) 200-207.

Ebata KT, Zhang X, Nagano \& MC 2005 Expression patterns of cell-surface molecules on male germ line stem cells during postnatal mouse development. Molecular Reproduction and Development 72(2) 171-181.

Foote RH 2002 The history of artificial insemination: Selected notes and notables. Iournal of Animal Science 80 1-10.

Geary TW, Whittier JC, Downing ER, LeFever DG, Silcox RW, Holland MD, Nett TM, \& Niswender GD 1998 Pregnancy rates of postpartum beef cows that were synchronized using Syncro-Mate-B or the Ovsynch protocol. Journal of Animal Science 76 1523-1527.

Herrid M, Vignarajan S, Davey R, Dobrinski I \& Hill JR 2006 Successful transplantation of bovine testicular cells to heterologous recipients. Reproduction 132(4) 617-624.

Izadyar F, Den Ouden K, Stout TA, Stout I, Coret J, Lankveld DP, Spoormakers TJ, Colenbrander B, Oldenbroek JK, Van der Ploeg KD, Woelders H, Kal HB \& De Rooij DG 2003 Autologous and homologous transplantation of bovine spermatogonial stem cells. Reproduction 126 765-774.

Kanatsu-Shinohara M, Toyokuni S \& Shinohara T 2004 CD9 is a surface marker on mouse and rat male germline stem cells. Biology of Reproduction $7070-75$.
Kanatsu-Shinohara M, Muneto T, Lee J, Takenaka M, Chuma S, Nakatsuji N, Horiuchi T \& Shinohara T 2007 Long-term culture of male germline stem cells from hamster testes. Biology of Reproduction 78(4) 611-617.

Kanatsu-Shinohara M, Ogonuki N, Inoue K, Miki H, Ogura A, Toyokuni S \& Shinohara T 2003 Long-term proliferation in culture and germline transmission of mouse male germline stem cells. Biology of Reproduction 69(2) 612-616.

Kanatsu-Shinohara $M$, Miki $H$, Inoue K, Ogonuki N, Toyokuni S, Ogura A \& Shinohara T 2005 Long-term culture of mouse male germline stem cells under serum-or feeder-free conditions. Biology of Reproduction 72(4) 985-991.

Kanatsu-Shinohara $M$, Inoue $K$, Ogonuki $N$, Miki $H$, Yoshida S, Toyokuni S, Lee J, Ogura A \& Shinohara T 2007 Leukemia inhibitory factor enhances formation of germ cell colonies in neonatal mouse testis culture. Biology of Reproduction 76(1) 55-62.

Kubota H, Avarbock MR \& Brinster RL 2004a Growth factors essential for self renewal and expansion of mouse spermatogonial stem cells. Proceedings of the National Academy of Sciences $10116489-16494$.

Kubota H, Avarbock MR \& Brinster RL 2004b Culture conditions and single growth factors affect fate determination of mouse spermatogonial stem cells. Biology of Reproduction $71722-731$.

Luo J, Megee S \& Dobrinski 12009 Asymmetric distribufion of UCH-L1 in spermatogonia is associated with maintenance and differentiation of spermatogonial stem cells. Journal of Cell Physiology 220 460-468.

Meng X, Lindahl M, Hyvönen ME, Parvinen M, de Rooij DG, Hess MW, Raatikainen-Ahokas A, Sainio K, Rauvala $H$, Lakso $M$, Pichel JG, Westphal $H$, Saarma $M$ \& Sariola H 2000 Regulation of cell fate decision of undifferentiated spermatogonia by GDNF. Science 287 1489-1493.

Mullaney BP \& Skinner MK 1992 Basic fibroblast growth factor (bFGF) gene expression and protein production during pubertal development of the seminiferous tubule: follicle-stimulating hormone-induced Sertoli cell bFGF expression. Endocrinology 131 2928-2934.

Nagano M, Avarbock MR \& Brinster RL 1999 Pattern and kinetics of mouse donor spermatogontal stem cell colonization in recipient testes. Biology of Reproduction 60 1429-1436.

Nagano M, Ryu BY, Brinster CJ, Avarbock MR, \& Brinster RL 2003 Maintenance of mouse male germ line stem cells in vitro. Biology of Reproduction $\mathbf{6 8}$ 2207-2214.

Oatley JM \& Brinster RL 2008 Regulation of spermatogonial stem cells self-renewal in mammals. Annual Review of Cell Developmental Biology 24 $263-286$.

Oatley JM \& Brinster RL 2006 Spermatogonial stem cells. Methods in Enzymology 419 259-282.

Oatley JM, Oatley MJ, Avarbock Tobias JW \& Brinster RL 2009 Colony stimulating factor 1 is an extrinsic 
stimulator of mouse spermatogonial stem cell self renewal. Development 136(7) 1191-1199.

Oatley JM, de Avila DM, Reeves JJ \& McLean DJ 2004a Testis tissue explant culture supports survival and proliferation of bovine spermatogonial stem cells. Biology of Reproduction 70 625-631.

Oatley IM, Reeves JJ \& McLean DJ 2004b Biological activity of cryopreserved bovine spermatogonial stem cells during in vitro culture. Biology of Reproduction 71 942-947.

Oatley IM, de Avila DM, McLean DJ, Griswold MD \& Reeves II 2002 Transplantation of bovine germinal cells into mouse testes. Journal of Animal Science 80(7) 1925-1931.

Perry GA, Smith MF, \& Patterson DJ 2002 Evaluation of a fixed-time artificial insemination protocol for postpartum suckled beef cows. Journal of Animal Science 80 3060-4.

Reding SR, Stepnoski AL, Cloninger EW \& Oatley IM 2010 THY 1 is a Conserved Marker of Undifferentiated Spermatogonia in the Pre-Pubertal Bull Testis. Reproduction 139 893-903.

Russell LD, Ettlin RA, Hikim APS, \& Clegg ED 1990 Histological and histocompatability evaluation of the testis, edn 1 Clearwater FL, Cache River Press.

Ryu BY, Kubota H, Avarbock MR \& Brinster RL 2005 Conservation of spermatogonial stem cell self-renewal signaling between mouse and rat. Proceedings of the National Academy of Sciences USA 102(40) 14302-14307.
Seandel M, James D, Shmelkov SV, Falciatori I, Kim J, Chavala S, Scherr DS, Zhang F, Torres R, Gale NW, Yancopoulos GD, Murphy A, Valenzuela DM, Hobbs RM, Pandolfi PP \& Rafii S 2007 Generation of functional multipotent adult stem cells from GPR125+ germline progenitors. Nature 449(7160) 346-350.

Sharpe R 1994 Regulation of spermatogenesis. In The Physiology of Reproduction, edn 2, pp 1363-1434. Eds E Knobil and JD Neill. New York: Raven Press.

Shinohara T, Avarbock MR \& Brinster RL 1999 B1- and $\alpha 6$-intergrin are surface markers and mouse spermatogonial stem cells. Proceedings of the National Academy of Sciences $965505-5509$.

Stockwell S, Herrid M, Davey R, Brownlee A, Hutton K \& Hill JR 2009 Microsatellite detection of donor-derived sperm DNA following germ cell transplantation in cattle. Reproduction, Fertility and Development 21(3) 462-468.

Tegelenbosch RAJ \& de Rooij DG 1993 A quantitative study of spermatogonial multiplication and stem cell renewal in the $\mathrm{C} 3 \mathrm{H} / 101 \mathrm{~F} 1$ hybrid mouse. Mutation Research $290193-200$.

Tadokoro Y, Yomogida K, Ohta H, Tohda A \& Nishimune Y 2002 Homeostatic regulation of germinal stem cell proliferation by the GDNF/FSH pathway. Mechanisms of Development 113(1) 29-39.

Yoshinaga K, Nishikawa S, Ogawa M, Hayashi S, Kunisada T, Fujimoto T \& Nishikawa S 1991 Role of $\mathrm{c}-\mathrm{kit}$ in mouse spermatogenesis: identification of spermatogonia as a specific site of c-kit expression and function. Development 113(2) 689-699. 